\section{Nanos2 suppresses meiosis and promotes male germ cell differentiation}

\author{
Atsushi Suzuki ${ }^{1}$ and Yumiko Saga ${ }^{1,2,3,4}$ \\ ${ }^{1}$ Department of Genetics, SOKENDAI, Mishima, Shizuoka \\ 411-8540, Japan; ${ }^{2}$ Division of Mammalian Development, \\ National Institute of Genetics, Mishima 411-8540, Japan; \\ ${ }^{3}$ Core Research for Evolutional Science and Technology \\ (CREST), Japan Science and Technology Corporation, \\ Minato-ku, Tokyo 105-0011, Japan
}

In mouse fetal gonads, retinoic acid (RA) induces meiosis in the female germ cells, whereas the male germ cells never enter meiosis due to Cyp26b1-mediated RA metabolism. We show here that Nanos2 plays critical roles in the differentiation of male germ cells. We find that Nanos2 maintains the suppression of meiosis by preventing Stra8 expression, which is required for premeiotic DNA replication, after Cyp26b1 is decreased. We also demonstrate that Nanos2 activates a male-specific genetic program, which is supported by the inhibition of meiosis and the induction of male-type differentiation in female germ cells following the forced expression of Nanos2.

Supplemental material is available at http://www.genesdev.org.

Received September 5, 2007; revised version accepted December 7, 2007.

Germ cells are highly specialized cells that transmit genetic information to subsequent generations. In mouse, primordial germ cells (PGCs) are segregated from the somatic cell lineages at early gastrulation. These PGCs, once generated, continue to proliferate and migrate through the somatic tissues to eventually reach the future gonads. Although PGCs are sexually bipotential at this migratory stage, they initiate sexual differentiation after their colonization of the gonads. During this process, the female germ cells enter into meiosis and proceed to the diplotene stage of meiotic prophase I, whereas the male germ cells initiate growth arrest at G1/G0 and undergo genome-wide DNA methylation and paternal imprinting (McLaren 2003; Allegrucci et al. 2005).

Recently, it has been reported that retinoic acid (RA) induces Stra8, an RA-responsive gene, in female germ cells, which then leads to meiotic initiation in the embryonic ovary. In contrast, RA signaling has been shown to be inhibited in fetal testes by Cyp26b1, a RA-metabolizing enzyme highly expressed in the somatic cells of the testes (Baltus et al. 2006; Bowles et al. 2006; Koubova et al. 2006). This event is thought to be a key step in the development of the male germ cell. However, it is still

[Keywords: Germ cell; meiosis; nanos; retinoic acid] ${ }^{4}$ Corresponding author.

E-MAIL ysaga@lab.nig.ac.jp; FAX 055-981-6828.

Article is online at http://www.genesdev.org/cgi/doi/10.1101/gad.1612708. unclear how male germ cells continue to escape from entry into meiosis throughout embryogenesis, given that Cyp26b1 expression gradually decreases during the later stages of embryonic development (Bowles et al. 2006; Koubova et al. 2006). In addition, although the mechanisms that trigger the sexual differentiation of the female germ cells are beginning to be revealed, the molecules that promote the male germ cell fate have remained largely unknown up until now.

\section{Results and Discussion}

\section{Nanos2 $2^{-/-}$male gonocytes enter meiosis}

Previously, we identified Nanos2 as a male PGC-specific gene involved in the maintenance of the male germ cells. In the absence of Nanos2, these cells undergo apoptosis from embryonic day 15.5 (E15.5), and most are subsequently lost by birth (Tsuda et al. 2003). This anti-apoptotic function of Nanos also appears to be conserved in several animals (Subramaniam and Seydoux 1999; Sato et al. 2007). However, during embryogenesis, mouse Nanos2 is specifically expressed only in the male germ cells after they have colonized the genital ridges (such cells are often referred to as male gonocytes), where they undergo cell cycle arrest at G1/G0. We speculated that Nanos2 would be involved in this mitotic arrest and, to test this hypothesis, we examined the mitotic activity of male gonocytes by double-immunostaining with antiphosphorylated histone $\mathrm{H} 3$ ( $\mathrm{pH} 3$, an M-phase marker) and TRA98 (a germ cell marker) (Fig. 1A-D). Although no significant $\mathrm{pH} 3$-positive cells could be detected in either Nanos2 $2^{+7-}$ or Nanos2 $2^{-/-}$male gonocytes at E14.5 (Fig. 1A,B), pH3-positive gonocytes became detectable from E15.5 in Nanos2 $2^{-/}$mouse embryos only (Fig. 1D). These data indicate that Nanos2-null male gonocytes appear to undergo cell cycle arrest normally at E14.5 but fail to maintain this arrest state and reinitiate proliferation from E15.5.

We speculated also that the Nanos2-null male gonocytes might have initiated meiosis in a similar manner to female germ cells, which do so at around embryonic stage E13.5. We therefore immunostained these cells with an antibody against synaptonemal complex protein 3 (Scp3). During meiosis, homologous chromosomes form a synaptonemal complex in which Scp3 is located along the paired meiotic chromosomes (Yuan et al. 2000). Our immunohistological analyses revealed that germ cells in the Nanos $2^{-\gamma-}$ testes abnormally express Scp3 at E15.5 (Fig. 1H), whereas very few cells were positive for this protein in the Nanos2 $2^{+/-}$male gonad (Fig. 1G). Nanos2-null male gonocytes at this embryonic stage present at both the inside and outside of the tubules due to the active migration of these cells from these structures, as we have reported previously (Tsuda et al. 2003). We found in our current analyses that these cells express Scp3 independently of their location. The Scp3 signals were very low and only marginally detectable in both Nanos2 $2^{+-}$and Nanos2 $2^{-/-}$testes at E14.5 (Fig. 1E,F), which suggested that male gonocytes initiate meiosis from E15.5 in the absence of Nanos2.

Upon the progression of normal meiosis, precursors of the synaptonemal complex show characteristic struc- 


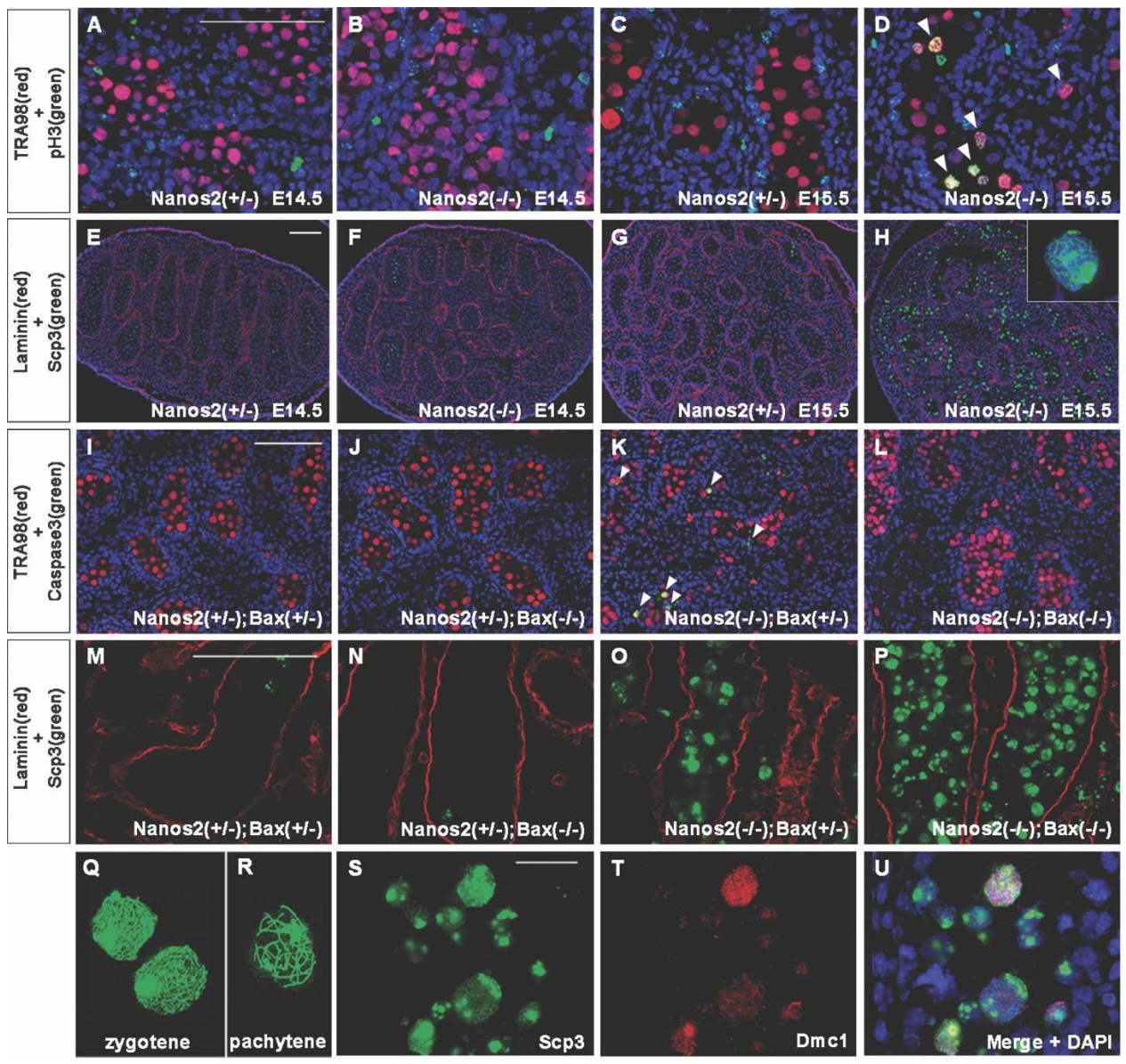

Figure 1. Nanos2-null male gonocytes enter meiosis. $(A-D)$ Embryonic testis samples prepared from Nanos2 $2^{+/-}$and Nanos2 $2^{-/-}$littermates at E14.5 and E15.5 were sectioned and immunostained with TRA98 (red) and anti-pH3 antibody (green). Arrowheads in $D$ indicate male gonocytes in $\mathrm{M}$ phase. $(E-H)$ Sections of testes from Nanos2 $2^{+-}$and Nanos2 $2^{-/-}$littermates at E14.5 and at E15.5 immunostained with anti-Scp3 (green) and anti-Laminin 1 (red) antibodies. The inset in $(H)$ shows a meiotic cell forming axial cores. $(I-P)$ Sections from Nanos $2^{+/-} \mathrm{Bax}^{+/-}, \mathrm{Nanos} 2^{+/-}$ $\mathrm{Bax}^{-1-}$, Nanos2 $2^{-/-} \mathrm{Bax}^{+-}$, and Nanos2 ${ }^{-/-} \mathrm{Bax}^{-/-}$E17.0 testes immunostained with TRA98 (red) and anti-activated-caspase 3 (green) (I-L), or anti-Laminin 1 (red) and anti-Scp3 (green) $(M-P)$ antibodies. Arrowheads in $K$ indicate apoptotic male gonocytes. $(Q, R)$ Chromosome spreads prepared from E17.0 Nanos $2^{-/-} \mathrm{Bax}^{-/-}$testes and immunostained with an anti-Scp3 antibody. There were many cells at the zygotene (Q) but few cells at the pachytene $(R)$ stage. ( $S-U$ ) Sections from Nanos2 ${ }^{-/-} \mathrm{Bax}^{-/-}$E17.0 testes immunostained with anti-Scp3 (green) and anti-Dmc1 (red) antibodies. DNA was labeled using the DAPI counterstain (blue). Bars: $A$ (for $A-D$ ), $E$ (for $E-H), I$ (for $I-L), M($ for $M-P$ ), $100 \mu \mathrm{m} ; S$, for $S-U$, $20 \mu \mathrm{m}$.

tures at the zygotene stage known as axial cores (Yuan et al. 2000). However, although the expression of Scp3 is highly up-regulated in Nanos $2^{-/-}$mice, we observed that almost all of the male gonocytes had morphologically abnormal nuclei and that only a few cells showed axial core formation, a clear indication of meiosis (Fig. $1 \mathrm{H}$, inset). We speculated therefore that Nanos2-null male gonocytes may have entered meiosis but subsequently may have undergone apoptotic cell death prior to axial core formation. To test this possibility, we knocked out the proapoptotic gene, Bax (Knudson et al. 1995; Stallock et al. 2003), together with Nanos2 to suppress apoptosis. We first confirmed that apoptosis was indeed suppressed in these double knockouts by assaying activated caspase3. In $\mathrm{Nanos} 2^{+/-} \mathrm{Bax}^{+/-}$and $\mathrm{Nanos} 2^{+/-}$ $\mathrm{Bax}^{-/-}$mice, there were no apoptotic signals detected (Fig. 1I,J). Nanos $2^{-/-} \mathrm{Bax}^{+/-}$mice showed numerous apoptotic male gonocytes (Fig. $1 \mathrm{~K}$ ), similar to Nanos $2^{-/-}$ mice (Tsuda et al. 2003). However, such apoptotic signals were undetectable in the Nanos $2^{-/} \mathrm{Bax}^{-/-}$background (Fig. 1L). In these double knockouts, many male gonocytes formed axial cores (Fig. 1P), and analysis of chromosome preparations showed that some of these are at the zygotene stage (Fig. 1Q), whereas others were at early pachytene stage (Fig. 1R) and show distinct threads representative of paired chromosomes. Moreover, these cells also expressed Dmc1, a meiosis-specific mammalian RecA homolog and a marker of meiotic cells from the leptotene to zygotene stages (Fig. 1S-U; Pittman et al. 1998; Chuma and Nakatsuji 2001). In contrast to these findings, little or no expression of Scp3 was observed in either Nanos $2^{+/-} \mathrm{Bax}^{+/-}$or Nanos $2^{+/-}$ $\mathrm{Bax}^{-/-}$mice (Fig. 1M,N), and the Nanos2 $2^{-/-} \mathrm{Bax}^{+/-}$male gonocyte phenotype (Fig. 1O) was similar to that of the Nanos2-single-null mouse (Fig. 1H). Hence, our current data indicate that one of the functions of Nanos2 is to block the male gonocytes from entry into meiosis, which might be the cause of the observed apoptotic response in the knockout animals. However, we cannot at present exclude the possibility that apoptosis is induced independently of abnormal meiosis in these cells. 


\section{The relationship between Nanos2 and $R A$ signaling}

Our initial data raise two key questions: (1) What is the mechanism by which Nanos2 suppresses meiosis in male gonocytes, and (2) what is the underlying reason for the meiotic entry delay in Nanos2-null male gonocytes compared with their female counterparts? RA signaling is known to be responsible for the induction of germ cell meiosis in the developing ovary, but is inhibited in the fetal testes by Cyp26b1. It has also been reported that abnormal meiosis is induced in Cyp26b1-null male gonocytes. These cells fail to suppress RA signaling, which results in the up-regulation of Stra8, leading to meiotic entry with a similar timing to female meiosis from E13.5 (Baltus et al. 2006; Bowles et al. 2006; Koubova et al. 2006; MacLean et al. 2007).

To evaluate the functions of Nanos 2 in the context of RA signaling, we first examined the Stra8 expression levels in Nanos2-null male gonocytes. Although this protein was suppressed in normal male gonocytes, an expected up-regulation was observed for Stra8 in E15.5 Nanos2-null male gonocytes (Fig. 2A-F). We next compared the level and timing of this up-regulation between Nanos2-null testes, wild-type embryonic ovaries, and Cyp26b1-null testes by real-time RT-PCR. In the embryonic ovaries, the Stra8 expression levels were transiently
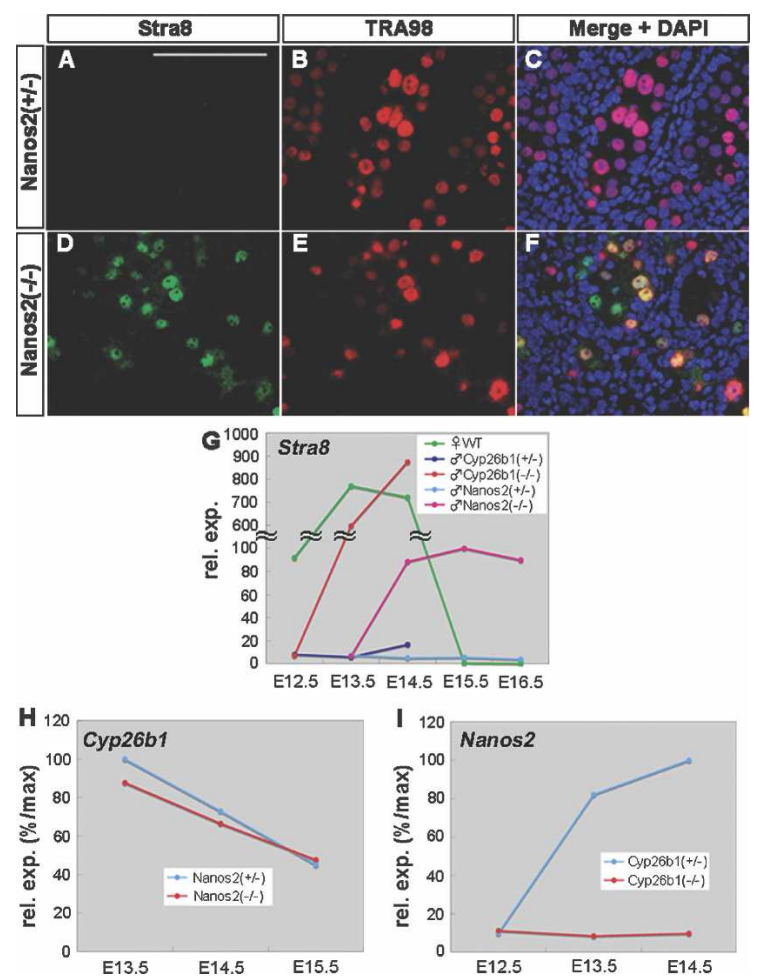

Figure 2. The relationship between Nanos 2 and RA signaling. (AF) Sections were prepared from E15.5 testes of $\mathrm{Nanos}^{+/-}$and Nanos $2^{-/-}$littermates, and were immunostained with TRA98 (red) and anti-Stra8 (green) antibodies. DNA was labeled with DAPI (blue). Bar: $A$ (for $A-F$ ), $100 \mathrm{um}$. $(G)$ Real-time RT-PCR analyses of Stra8 in E12.5-E16.5 wild-type ovaries (green), and in E12.5-E14.5 testes of Cyp26b1 $1^{+/-}$(dark blue) and Cyp26b1 $1^{-/-}$(orange) littermates, and also in E13.5-E16.5 testes of Nanos2 $2^{+-}$(blue) and Nanos2-/(red) littermates. $(H, I)$ Real-time RT-PCR analyses of Cyp26b1 (H) in E13.5-E15.5 testes of Nanos $2^{+/-}$and Nanos $2^{-/-}$littermates, and of Nanos2 $(I)$ in E12.5-E14.5 testes of Cyp26b1 $1^{+-}$and Cyp26b1 $1^{-/-}$littermates. up-regulated from E13.5 to E14.5, and then down-regulated to undetectable amounts after E15.5 (Fig. 2G, green). Similarly, in the Cyp26b1-null testes, the upregulation of Stra8 was observed from E13.5 to E14.5 at levels comparable with the embryonic ovaries (Fig. 2G, orange). In contrast, Stra8 expression was very low in Nanos2-null testes up to E13.5 but found to be increased from E14.5 and was maintained at higher levels at least until E16.5 (Fig. 2G, red). Very little expression of this gene was evident in normal testes (Fig. 2G, dark blue and blue).

The up-regulation of Stra8 thus appears to be responsible for the induction of meiosis in Nanos2-null male gonocytes. However, the timing of this activation was delayed and the expression level was lower when compared with Cyp26b1-null testes (Fig. 2G, orange and red). This could be due to the normal expression of Cyp26b1 in Nanos2-null testes as revealed by our real-time RTPCR analyses (Fig. 2H). Even after E14.5, Cyp26b1 was still present at a detectable level in Nanos2 $2^{-/-}$testes, so that Stra 8 expression is likely to be suppressed to some degree. However, we surmised that Nanos 2 must be required for the complete suppression of Stra8, which was expressed in Nanos2-null male gonocytes at sufficient levels to induce meiosis. This observation prompted us to examine the expression of Nanos2 in the Cyp26b1null testes. Interestingly, we found that Nanos2 expression was not induced in the absence of Cyp26b1 (Fig. 2I), which also accounts for the continuous up-regulation of Stra8 in Cyp26b1-null testes. Hence, Cyp26b1-mediated RA metabolism might set the conditions under which Nanos2 expression can occur. Taken together, we conclude from our data that Cyp26b1 alone is not sufficient to prevent male germ cell meiosis and that Nanos 2 in the male gonocytes is required for the complete suppression of Stra8 after E14.5.

\section{Meiosis is inhibited in Nanos2-expressing female germ cells}

To further clarify the function of Nanos2 in the suppression of meiosis, we attempted to ectopically express this gene in female embryonic germ cells by generating a transgenic mouse line. We initially established a conditional transgenic mouse line, CAG-loxP-CAT-loxP-Flagtagged-Nanos2, that is capable of expressing FlagNanos2 under the control of the CAG promoter after excision of the floxed CAT gene by Cre recombinase. We utilized Nanos3-Cre knock-in mice to induce FlagNanos2 expression in the germ cell lineage of the transgenic progeny. By crossing with Nanos3-Cre mice, Cre/ loxP-specific CAT excision occurs in Nanos3-expressing cells, and this would have been expected to result in the induction of Flag-tagged Nanos2 expression in the germ cell lineage. Nanos3-cre activity was detected at E7.75 by $\beta$-gal reporter activity, and this signal was restricted in the germ cells during both the migration stage and after their entry into the gonad (our unpublished observation). Unfortunately, however, Cre recombinase was found to have activated in only a portion of the PGCs in this Nanos3-cre line (the efficiency is $<25 \%$ ), but we could confirm an appreciable level of Flag-tagged Nanos 2 expression in some populations of female germ cells in the double transgenic mice, CAG-CAT-Flag-Nanos2/ Nanos3-cre (Fig. 3A,B). Flag-Nanos 2 could be detected in the cytoplasm of these female germ cells, and a similar 


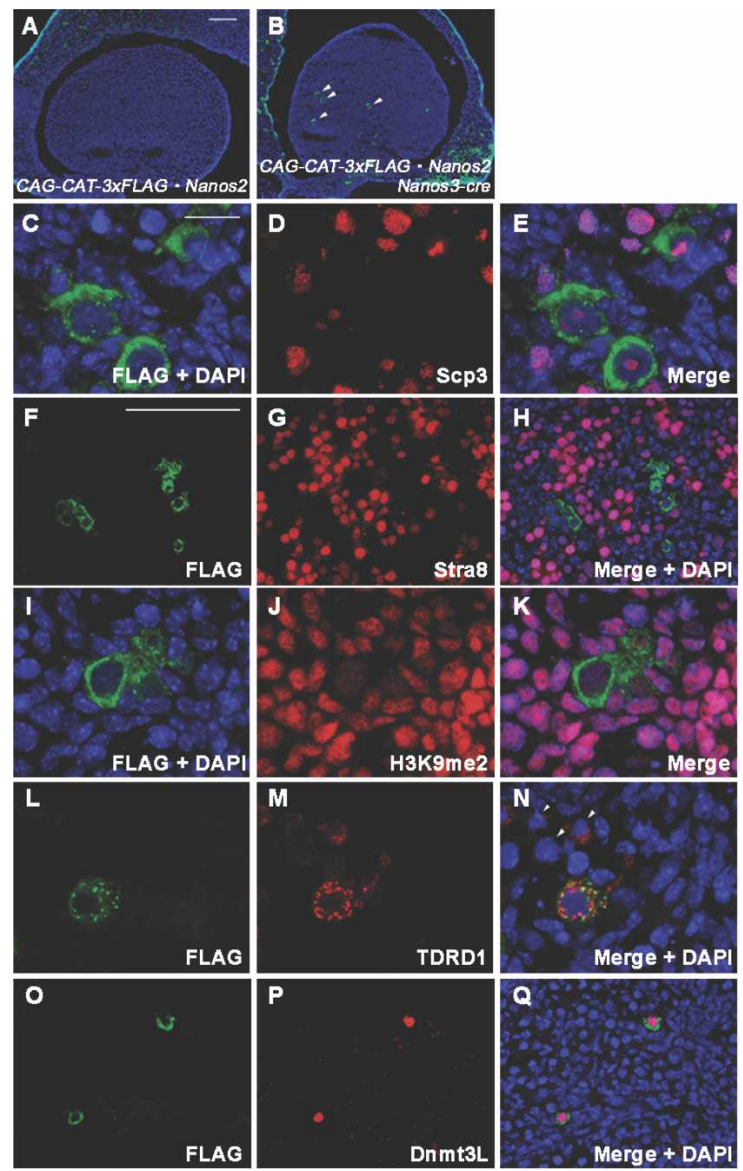

Figure 3. The forced expression of Nanos 2 prevents meiosis and induces male characteristics in female embryonic germ cells. $(A-E)$ Ovaries from control $(A)$ and Nanos2-expressing $(B-E)$ littermates at E16.5 were sectioned and immunostained with anti-Flag (green) and anti-Scp3 (red) antibodies. Arrowheads in $B$ indicate Nanos2-positive female germ cells. $(F-H)$ Sections were prepared from E13.5 ovaries of Nanos2-expressing mice and were immunostained with anti-Flag (green) and anti-Stra8 (red) antibodies. (I-Q) Sections were prepared from E16.5 ovaries of Nanos2-expressing mice and were immunostained with anti-Flag (green) $(I, L, Q)$ and anti-dimethylated histone H3K9 $(J)$, anti-TDRD1 $(M)$, or anti-Dnmt3L $(P)$ antibodies. Arrowheads in $N$ indicate Nanos2-negative female germ cells. DNA was labeled by DAPI counterstaining (blue). Bars: $A$ (for $A, B$ ), $F$ (for $F-H, O-Q, I, 100 \mu \mathrm{m}$; $C$ for $C-E, I-N), 20 \mu \mathrm{m}$.

expression pattern was also detectable by anti-Nanos2 antibody staining (Supplemental Fig. S1).

We examined whether meiosis is suppressed in Nanos2-expressing female germ cells by performing antiScp3 staining. In normal female germ cells, Scp3 signals are detectable from E12.5, and axial core formation begins at E14.5. The synaptonemal complex is then formed by E16.5 (Supplemental Fig. S2). However, in the Nanos2-expressing female germ cells, we observed that the formation of these complexes was inhibited, and that small nuclear dots corresponding to Scp3 signals were evident only at E16.5 (Fig. 3C-E). We next assessed whether Nanos2 had any suppressive effects on Stra8, an essential factor for the progression of meiosis (Baltus et al. 2006). The expression of Stra8 is induced in female germ cells from E12.5, transiently increases at E13.514.5, and decreases thereafter (Fig. 2G; Menke et al. 2003). However, we could not detect Stra8 in female germ cells expressing Nanos2 at E13.5 (Fig. 3F-H), indicating that Nanos2 can block meiosis by suppressing Stra8 expression.

The mechanisms underlying the suppression of Stra8 by Nanos 2 are currently under investigation in our laboratory. However, we found in our current experiments that the transcription of Stra8 is up-regulated in Nanos2 ${ }^{-/-}$male gonocytes and down-regulated in Nanos2-expressing female germ cells (Supplemental Fig. S3). Therefore, given that Nanos2 is an RNA-binding protein localized in the cytoplasm, the up- or down-regulation of Stra8 transcription would need to be an indirect effect of Nanos2.

\section{Nanos2 induces male-type differentiation in female germ cells}

In addition to the suppression of meiosis, we observed an enlarged nuclear morphology in Nanos2-expressing female germ cells and a reduction in their heterochromatic regions in a similar manner to male gonocytes (Fig. 3C). This prompted us to speculate that these female germ cells might have adopted a male germ cell fate. It has been shown that the chromatin structure in the germ cells changes according to their sexual differentiation, and this can be monitored by the methylation status of the histone proteins. One of the markers associated with this is the dimethylation of histone H3-Lys9 (H3K9me2) (Seki et al. 2005). In wild-type male gonocytes, decreased levels of methylation are evident in comparison with the surrounding somatic cells (Supplemental Fig. S4A-F), but we never observed this in Nanos2-null male gonocytes (Supplemental Fig. S4G-J). In Nanos2-expressing female germ cells, a decrease in $\mathrm{H} 3 \mathrm{~K} 9 \mathrm{me} 2$ immunoreactivity was also observed (Fig. 3I-K). To further characterize these Nanos2-positive female germ cells, we performed immunostaining analysis of two molecules that are normally expressed in the male gonocytes only. Tudor domain-containing 1 (TDRD1) is a component of chromatoid bodies/nuages in male germ cells. It has been reported that the expression of this protein increases from E13.5 in male gonocytes, but is maintained at much lower levels in female germ cells /Chuma et al. 2003). As reported previously, we occasionally observed weaker signals in the Nanos2-negative female germ cells, but TDRD1 was detectable as discernible granules in the cytoplasm of Nanos2-positive female germ cells (Fig. 3L-N). Dnmt3L is implicated in genomic imprinting and is expressed in male gonocytes from E14.5 to E18.5, but its expression is initiated only after birth in female germ cells (Bourc'his et al. 2001; Bourc'his and Bestor 2004; Sakai et al. 2004). Strikingly, we observed a strong nuclear expression of Dnmt3L only in Nanos2expressing female germ cells (Fig. 3O-Q).

It is noteworthy that Nanos 2 expression was induced by Nanos3-cre activity in the transgenic mice, resulting in the continuous expression of Nanos 2 from as early as E7.75 (our unpublished data). Therefore, the early expression of Nanos 2 might account for the fate change of female germ cells. However, when Nanos 2 expression was induced under the direct control of the Oct3/4- $\Delta \mathrm{PE}$ enhancer, the resulting female mice produced normal oocytes and were fertile (Suzuki et al. 2007). This must be due to the property of the Oct3/4- $\Delta \mathrm{PE}$ enhancer, which is known to become inactive in the female germ cells after E13.5. Therefore, we conclude that the phenotype we 

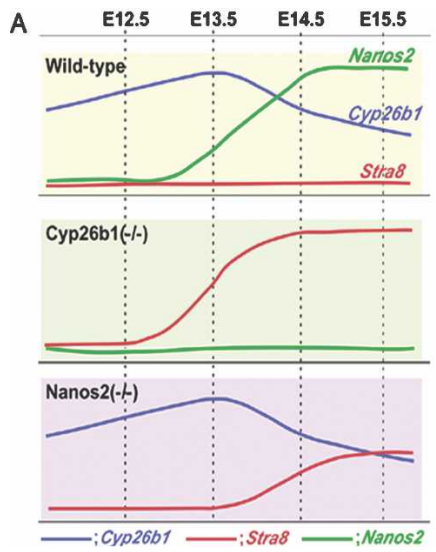

B

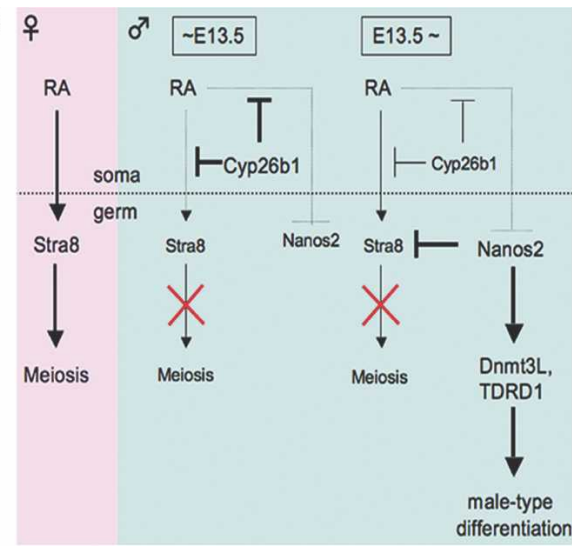

Figure 4. Expression profiles of critical factors in the embryonic testes and a hypothetical model for the sexual differentiation of mouse germ cells. (A) Expression patterns for Cyp26b1 (blue), Stra8 (red), and Nanos2 (green) in E12.5-E15.5 wild-type, Cyp26b1-null, and Nanos2-null testes. In wild-type mice, Cyp26b1 is increased until E13.5 and gradually reduces thereafter (Fig. 2H; Bowles et al. 2006), whereas Nanos2 expression starts at around E13.5 and Stra 8 is not induced. In Cyp26b1-null mice, Stra8 is up-regulated at E13.5 but is not induced until E14.5 in Nanos2-null mice, possibly due to the effects of Cyp26b1 (Fig. 2G). (B, left) RA signaling triggers meiotic initiation via the induction of Stra8 expression in female germ cells (pink). (Middle) In embryonic testes, Cyp26b1 inhibits RA signaling, which suppresses the entry of male gonocytes into meiosis and may provide the required conditions for the expression of Nanos2 until E13.5 (blue). (Right) After Cyp26b1 expression is decreased, Nanos2 inhibits meiotic initiation via suppression of Stra8 expression and induces male-type differentiation, which is indicated by the presence of the Dnmt3L and TDRD1 (blue).

observed in the Nanos2-expressing female germ cells is due to Nanos2 expression after E13.5, and not due to the expression of this protein in the earlier stages.

These findings suggest, therefore, that Nanos 2 converts female germ cells to male-type germ cells under our experimental conditions. In agreement with this possibility, the expression of TDRD1 and Dnmt3L was lowered or not induced in Nanos2-null male gonocytes (Supplemental Fig. S5), indicating that these cells cannot initiate normal male pathways in the absence of Nanos2. These findings, together with our results showing the ectopic induction of meiosis, suggested that the male gonocytes had acquired a female germ cell fate. Although we did not observe oocyte-like cells at any stage in the Nanos2/Bax-null testes, we did observe the up-regulation of genes that are required only for female germ cell development, even in the Nanos2 $2^{-/-}$testis at E16.5 (Supplemental Fig. S6). This suggests that Nanos2-null male gonocytes have become feminized. The number of germ cells began to reduce gradually after birth, and finally disappeared by $4 \mathrm{wk}$, even in the Nanos2/Baxdouble-null background (Supplemental Fig. S7). We reasoned from this that the germ cells are unable to retain their female characteristics because the surrounding somatic cells in the Nanos2-null testes are not themselves feminized.

\section{Hypothetical model for the sexual differentiation of mouse germ cells}

Based on our current results, we devised a working model for the sexual differentiation of mouse germ cells, which is outlined in Figure 4. Both Cyp26b1 and Nanos2-null mice show similar ectopic Stra8 expression phenotypes and the initiation of male gonocyte meiosis. However, the timing of the relevant events is different in these knockouts; in the Cyp26b1-null testes, Stra 8 induction occurs at E13.5, which is a similar stage to embryonic ovaries, but does not do so until E14.5 in the Nanos2-null testes (Fig. 4A). The difference in timing between Cyp26b1-null and Nanos2-null male gonocytes is due to different mechanisms operating therein (Fig. 4B). Somatically produced RA strongly induces Stras in female germ cells (Fig. 4B, left), which also occurs in Cyp26b1-null male gonocytes. In contrast, Cyp26b1 inhibits RA signaling in normal embryonic testes (Fig. 4B, middle). However, its expression is gradually reduced after E13.5, whereas the high levels of RA are maintained in the male embryonic mesonephros (Bowles et al. 2006). Nanos 2 then begins to be expressed at the point Cyp26b1 is down-regulated (Fig. 4B, right). That is why Stra8 is induced in Nanos2-null male gonocytes after E14.5.

It is likely that male gonocytes do not express Nanos2 if they are exposed to RA before the stage at which this gene is normally expressed, as revealed by its lack of induction in Cyp26b1-null testes (Fig. 2I). This in turn indicates that the inhibition of RA signaling is required not only for suppression of Stra8 expression but also induction of Nanos2. Once Nanos2 is induced, a male germ cell-specific program is initiated, and the germ cells then acquire a masculine fate (Fig. 4B, right) even though RA is present. Our present study thus demonstrates that Nanos2 is a crucial factor in male germ cell development. Identifying the factors required for the induction of Nanos2 expression will be essential in future studies to elucidate the principal sexual determinants in male germ cells.

\section{Materials and methods}

See the Supplemental Material for additional details.

Knockout and transgenic mice

The Nanos2-knockout mouse lines have been established previously. The $B a x^{t m 1 S j k}$ mutant mouse was purchased from the Jackson Laboratory. The generation of the Nanos3-Cre knock-in mice will be described elsewhere (our unpublished data). The CAG-loxP-CAT-lox-Flag-taggedNanos2 transgenic mouse lines were established using a conventional transgenic technique.

Histological methods

For immunostaining, the gonads of both sexes were directly embedded in O.C.T. compound and frozen in liquid nitrogen. The samples were then sectioned and fixed in $4 \%$ PFA. After preincubation with 3\% skim milk, the sections were stained with each primary antibody and followed by the appropriate secondary antibody.

Real-time RT-PCR

Total RNA isolates from male gonads were prepared using an RNeasy Mini Kit (Qiagen). One microgram of total RNA was then used for cDNA synthesis by SuperScript III reverse transcriptase (Invitrogen). SYBR Premix Ex-Taq (Takara) was used for the real-time PCR experiments according to the manufacturer's instructions.

\section{Acknowledgments}

We thank Y. Nishimune for generously providing the TRA98 antibody, S. Chuma and N. Nakatsuji for donating the anti-Scp3 and anti-TDRD1 
antibodies, S. Yamanaka for the anti-Dnmt3L antibody, P. Chambon for the anti-Stra8 antibody, and H. Hamada for providing the Cyp26b1 mutant male gonads. This work was supported in part by Grants-in-aid for a Core Research for Evolutional Science and Technology (CREST) project from the Japan Science and Technology Cooperation, and also by grants by the National BioResource Project (NBRP) and of the Genome Network Project from the Ministry of Education, Culture, Sports, Science and Technology, Japan.

\section{References}

Allegrucci, C., Thurston, A., Lucas, E., and Young, L. 2005. Epigenetics and the germline. Reproduction 129: 137-149.

Baltus, A.E., Menke, D.B., Hu, Y.C., Goodheart, M.L., Carpenter, A.E., de Rooij, D.G., and Page, D.C. 2006. In germ cells of mouse embryonic ovaries, the decision to enter meiosis precedes premeiotic DNA replication. Nat. Genet. 38: 1430-1434.

Bourc'his, D. and Bestor, T.H. 2004. Meiotic catastrophe and retrotransposon reactivation in male germ cells lacking Dnmt3L. Nature 431: 96-99.

Bourc'his, D., Xu, G.L., Lin, C.S., Bollman, B., and Bestor, T.H. 2001. Dnmt3L and the establishment of maternal genomic imprints. Science 294: 2536-2539.

Bowles, J., Knight, D., Smith, C., Wilhelm, D., Richman, J., Mamiya, S., Yashiro, K., Chawengsaksophak, K., Wilson, M.J., Rossant, J., et al. 2006. Retinoid signaling determines germ cell fate in mice. Science 312: $596-600$.

Chuma, S. and Nakatsuji, N. 2001. Autonomous transition into meiosis of mouse fetal germ cells in vitro and its inhibition by gp130-mediated signaling. Dev. Biol. 229: 468-479.

Chuma, S., Hiyoshi, M., Yamamoto, A., Hosokawa, M., Takamune, K., and Nakatsuji, N. 2003. Mouse Tudor Repeat-1 (MTR-1) is a novel component of chromatoid bodies/nuages in male germ cells and forms a complex with snRNPs. Mech. Dev. 120: 979-990.

Knudson, C.M., Tung, K.S., Tourtellotte, W.G., Brown, G.A., and Korsmeyer, S.J. 1995. Bax-deficient mice with lymphoid hyperplasia and male germ cell death. Science 270: 96-99.

Koubova, J., Menke, D.B., Zhou, Q., Capel, B., Griswold, M.D., and Page, D.C. 2006. Retinoic acid regulates sex-specific timing of meiotic initiation in mice. Proc. Natl. Acad. Sci. 103: 2474-2479.

MacLean, G., Li, H., Metzger, D., Chambon, P., and Petkovich, M. 2007. Apoptotic extinction of germ cells in testes of Cyp26b1 knockout mice. Endocrinology 148: 4560-4567.

McLaren, A. 2003. Primordial germ cells in the mouse. Dev. Biol. 262: $1-15$.

Menke, D.B., Koubova, J., and Page, D.C. 2003. Sexual differentiation of germ cells in XX mouse gonads occurs in an anterior-to-posterior wave. Dev. Biol. 262: 303-312.

Pittman, D.L., Cobb, J., Schimenti, K.J., Wilson, L.A., Cooper, D.M., Brignull, E., Handel, M.A., and Schimenti, J.C. 1998. Meiotic prophase arrest with failure of chromosome synapsis in mice deficient for Dmc1, a germline-specific RecA homolog. Mol. Cell 1: 697-705.

Sakai, Y., Suetake, I., Shinozaki, F., Yamashina, S., and Tajima, S. 2004. Co-expression of de novo DNA methyltransferases Dnmt3a2 and Dnmt3L in gonocytes of mouse embryos. Brain Res. Gene Expr. Patterns 5: 231-237.

Sato, K., Hayashi, Y., Ninomiya, Y., Shigenobu, S., Arita, K., Mukai, M., and Kobayashi, S. 2007. Maternal Nanos represses hid/skl-dependent apoptosis to maintain the germ line in Drosophila embryos. Proc. Nat1. Acad. Sci. 104: 7455-7460.

Seki, Y., Hayashi, K., Itoh, K., Mizugaki, M., Saitou, M., and Matsui, Y. 2005. Extensive and orderly reprogramming of genome-wide chromatin modifications associated with specification and early development of germ cells in mice. Dev. Biol. 278: 440-458.

Stallock, J., Molyneaux, K., Schaible, K., Knudson, C.M., and Wylie, C. 2003. The pro-apoptotic gene Bax is required for the death of ectopic primordial germ cells during their migration in the mouse embryo. Development 130: 6589-6597.

Subramaniam, K. and Seydoux, G. 1999. nos-1 and nos-2, two genes related to Drosophila nanos, regulate primordial germ cell development and survival in Caenorhabditis elegans. Development 126: 4861-4871.

Suzuki, A., Tsuda, M., and Saga, Y. 2007. Functional redundancy among
Nanos proteins and a distinct role of Nanos2 during male germ cell development. Development 134: 77-83.

Tsuda, M., Sasaoka, Y., Kiso, M., Abe, K., Haraguchi, S., Kobayashi, S., and Saga, Y. 2003. Conserved role of nanos proteins in germ cell development. Science 301: 1239-1241.

Yuan, L., Liu, J.G., Zhao, J., Brundell, E., Daneholt, B., and Hoog, C. 2000. The murine SCP3 gene is required for synaptonemal complex assembly, chromosome synapsis, and male fertility. Mol. Cell 5: 73-83. 


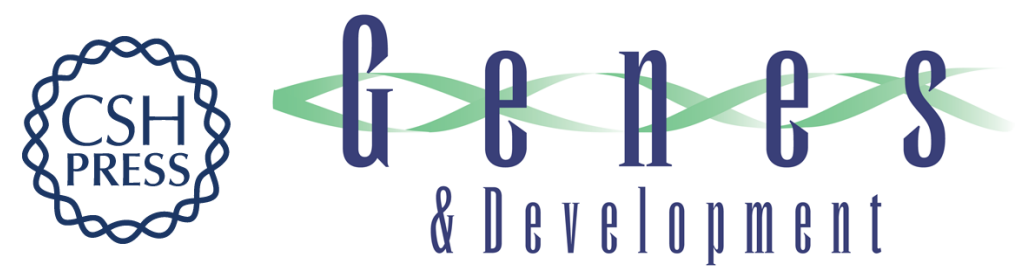

\section{Nanos2 suppresses meiosis and promotes male germ cell differentiation}

Atsushi Suzuki and Yumiko Saga

Genes Dev. 2008, 22:

Access the most recent version at doi:10.1101/gad.1612708

\section{Supplemental http://genesdev.cshlp.org/content/suppl/2008/01/29/22.4.430.DC1 Material}

References This article cites 21 articles, 10 of which can be accessed free at: http://genesdev.cshlp.org/content/22/4/430.full.html\#ref-list-1

\section{License}

Email Alerting

Receive free email alerts when new articles cite this article - sign up in the box at the top Service right corner of the article or click here.

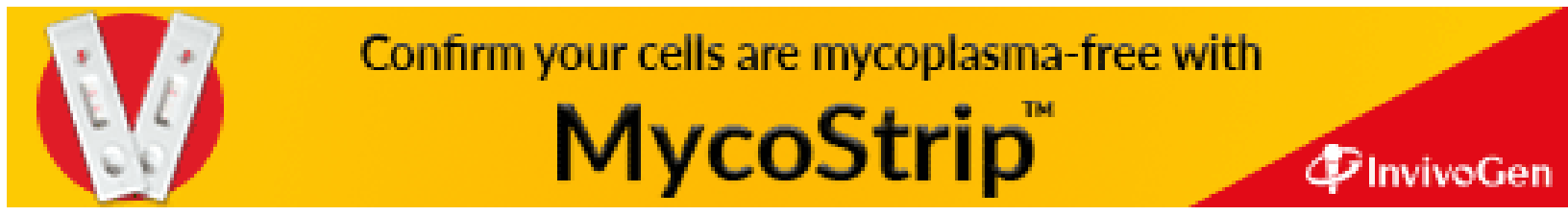

